\title{
ON THE DIMENSION OF MODULES AND ALGEBRAS, IV DIMENSION OF RESIDUE RINGS OF HEREDITARY RINGS
}

\author{
SAMUEL EILENBERG, HIROSI NAGAO and TADASI NAKAYAMA
}

A ring (with unit element) $A$ is called semi-primary ${ }^{1 \text { ) }}$ if it contains a nilpotent two-sided ideal $N$ such that the residue ring $\Gamma=1 / N$ is semi-simple (i.e. $1 . \operatorname{gl} \cdot \operatorname{dim} \Gamma=$ r.gl. $\operatorname{dim} \Gamma=0) . \quad N$ is then the (Jacobson) radical of $A$. Auslander [1] has shown that if $A$ is semi-primary then

1.gl. $\operatorname{dim} \Lambda=$ r.gl. $\operatorname{dim} A=1 \cdot \operatorname{dim}_{\Lambda} \Gamma=1+1 \cdot \operatorname{dim}_{\Delta} N$.

The common value is denoted by $\operatorname{gl} \operatorname{dim} A$. On the other hand, for any ring $A$ the following conditions are equivalent: (a) $1 . \mathrm{gl}$. dim $A \leqq 1$, (b) each left ideal in $A$ is projective, (c) every submodule of a projective left 1 -module is projective. Rings satisfying conditions (a)-(c) are called hereditary. For integral domains the notions of "hereditary ring" and "Dedekind ring" coincide.

If $a$ is a two-sided ideal in $A$, there is in general very little relation between 1. $g 1 . \operatorname{dim} A$ and $1 . \mathrm{gl} \operatorname{dim}(\Lambda / \mathfrak{a})$; it was however proved, substantially, in Eilenberg-Ikeda-Nakayama [4] that if $A$ is semiprimary and $a$ is contained in the radical $N$ then

$$
\text { gl. } \operatorname{dim} A \leqq \operatorname{gl} \operatorname{dim}(A / a)+1 \cdot \operatorname{dim}_{\Lambda}(A / a) .
$$

Now, we show in $\S 1$, of the present note, that if $A$ is hereditary and the sequence $a^{i}(i=1,2, \ldots)$ becomes constant then $\operatorname{gl} \operatorname{dim}(1 / a)<\infty$. Thus if $\Lambda$ is hereditary and semi-primary then $\operatorname{gl} \operatorname{dim}(i / a)<\infty$, and we are able to give, in $\S 2$, rather precise estimates for $\operatorname{gl} \operatorname{dim}(A / \mathfrak{a})$.

In $\S 3$ we show by examples that the above results are the best possible; thus for each pair $(m, n)$ with $0<m \leqq \infty, 0<n \leqq \infty,(m, n) \neq(1, \infty)$ we construct a semi-primary ring $\Lambda$ and a two-sided ideal a of $\Lambda$ such that gl. $\operatorname{dim} A=m$, gl. $\operatorname{dim}(A / \mathfrak{a})=n$.

Received October 15, 1955.

1) Our notion of "semi-primary" does not coincide with "halbprimär" of Deuring, Algebren, Ergebn. Math. 
In $\S 4$ we consider an algebra $A$ over a field $K$ with $(\Lambda: K)<\infty$. Here we consider the number $\operatorname{dim} A$ which is the highest integer $n$ such that the cohomology group $H^{n}(\Lambda, A)$ is not zero for some two-sided $\Lambda$-module $A$. If no such integer exists then $\operatorname{dim} A=\infty$. We show that results analogous to those of $\S \S 2-3$ hold.

\section{$\S 1$. Residue rings of hereditary rings}

Proposition 1. If $A$ is a ring (with unit element), a a two-sided ideal in $A$ and $B$ a projective left $A$-module, then the loft $(A / a)$-module $B / a B$ is projective.

Proof. The conclusion is trivial if $B=\Lambda$. Thus by a direct sum argument it is also valid if $B$ is $A$-free. Again by a direct sum argument, the conclusion follows for $B$ projective.

Proposition 2. Let $A$ be a ring, a a nilpotent left ideal in $A, A$ a left $\Lambda$ module and $B$ a submodule of $A$. If $A=B+a A$ then $A=B$.

Proof. Assume $C=A / B \neq 0$. Then since $a$ is nilpotent we have $a C \neq C$. But $a C=(B+a A) / B$. Thus $B+a A \neq A$, a contradiction.

Proposition 3. Let $A$ be a hereditary ring, let a be a two-sided ideal in $\Lambda$ and $b$ a left ideal containing $a$. The following conditions are equivalent (for $i \geq 0)$;

$$
\begin{gathered}
1 \cdot \operatorname{dim}_{\Lambda / \mathfrak{a}}(\mathfrak{b} / \mathfrak{a}) \leqq 2 i, \\
\mathfrak{a}^{i} \mathfrak{b} / \mathfrak{a}^{i+1} \text { is }(\Lambda / \mathfrak{a}) \text {-projective, }
\end{gathered}
$$

(iii) there exists a left ideal c in $A$ such that

$$
\mathfrak{c}+\mathfrak{a}^{i+1}=\mathfrak{a}^{i} \mathfrak{b}, \quad c \cap \mathfrak{a}^{i+1}=\mathfrak{a}^{i+1} \mathfrak{b} .
$$

Similarly, the following conditions are equivalent (for $i>0$ ):

$$
\begin{gathered}
1 . \operatorname{dim}_{\Lambda / \mathfrak{a}}(\mathfrak{b} / \mathfrak{a}) \leqq 2 i-1, \\
\mathfrak{a}^{i} / \mathfrak{a}^{i} \mathfrak{b} \text { is }(1 / \mathfrak{a}) \text {-projective, }
\end{gathered}
$$

(iii') there exists a left ideal $c$ in $A$ such that

$$
c+a^{i} \mathfrak{b}=a^{i}, \quad c \cap a^{i} b=a^{i+1} .
$$

Proof. Since in the ring $A$ every left ideal $c$ is $A$-projective, it follows from Prop. 1 that $c / a c$ is $(A / \mathfrak{a})$-projective. Consider the sequence of ideals

$$
\ldots \subset a^{i+1} \mathfrak{b} \subset a^{i+1} \subset a^{i} \mathfrak{b} \subset a^{i} \subset \ldots \subset a^{2} \subset a \mathfrak{b} \subset a \subset b \text {. }
$$


The inclusion maps induce an exact sepuence

$$
\ldots \rightarrow \mathfrak{a}^{i} \mathfrak{b} / \mathfrak{a}^{i+1} \mathfrak{b} \rightarrow \mathfrak{a}^{i} / \mathfrak{a}^{i+1} \rightarrow \mathfrak{a}^{i-1} \mathfrak{b} / \mathfrak{a}^{i} \mathfrak{b} \rightarrow \ldots \rightarrow \mathfrak{a} / \mathfrak{a}^{2} \rightarrow \mathfrak{b} / \mathfrak{a b} \rightarrow \mathfrak{b} / \mathfrak{a} \rightarrow 0
$$

which is a projective resolution of $b / a$ as a $(A / \mathfrak{a})$-module. Now replace the term $\mathfrak{a}^{i} \mathfrak{b} / \mathfrak{a}^{i+1} \mathfrak{b}$ (occuring in degree $2 i$ ) by the image of $\mathfrak{a}^{i} \mathfrak{b} / \mathfrak{a}^{i+1} \mathfrak{b} \rightarrow \mathfrak{a}^{i} / \mathfrak{a}^{i+1}$ i.e. by $\mathfrak{a}^{i} \mathfrak{b} / \mathfrak{a}^{i+1}$, and replace the higher terms by zero. There results an exact sequence which implies the equivalence of (i) and (ii). Condition (iii) is simply an expression of the fact (equivalent with (ii)) that the exact sequence of $(A / \mathfrak{a})$ modules

$$
0 \rightarrow \mathfrak{a}^{i+1} / \mathfrak{a}^{i+1} \mathfrak{b} \rightarrow \mathfrak{a}^{i} \mathfrak{b} / \mathfrak{a}^{i+1} \mathfrak{b} \rightarrow \mathfrak{a}^{i} \mathfrak{b} / \mathfrak{a}^{i+1} \rightarrow 0
$$

splits.

The second part is proved similarly by replacing the term $a^{i} \mathfrak{b} / a^{i+1}$ by $a^{i} / a^{i} \mathfrak{b}$.

Proposition 4. Let $A$ be a hereditary ring and $\mathfrak{b}$ a nilpotent two-sided ideal in $A$. Then for $k>1$ we have:

$$
\begin{array}{ll}
\text { 1. } \operatorname{dim}_{\Delta / \mathfrak{b}^{k}}\left(\mathfrak{b} / \mathfrak{b}^{k}\right) \leqq 2 i \Leftrightarrow \mathfrak{b}^{k(i+1)}=0, & \text { for } i \geqslant 0 ; \\
\text { 1. } \operatorname{dim}_{\Delta / \mathfrak{b}^{k}}\left(\mathfrak{b} / \mathfrak{b}^{k}\right) \leqq 2 i-1 \Leftrightarrow \mathfrak{b}^{k i+1}=0, & \text { for } i>0 .
\end{array}
$$

Proof. We apply Prop. 3 with $a=b^{k}$. Condition (iii) then reads $c+b^{k(i+1)}$ $=\mathfrak{b}^{k i+1}, \mathfrak{c} \cap \mathfrak{b}^{k(i+1)}=\mathfrak{b}^{k(i+1)+1}$. Since $k>1$ we have $\mathfrak{b}^{k(i+1)} \subset \mathfrak{b b}^{k i+1}$. Thus by Prop. 2 the first condition becomes $c=b^{k i+1}$. The second condition then becomes $b^{k(i+1)}$ $=\mathfrak{b}^{k(i+1)+1}$. Since $\mathfrak{b}$ is nilpotent, this is equivalent with $\mathfrak{b}^{k(i+1)}=0$.

Condition (iii') reads: $\mathfrak{c}+\mathfrak{b}^{k i+1}=\mathfrak{b}^{k i}, \mathfrak{c} \cap \mathfrak{b}^{k i+1}=\mathfrak{b}^{k(i+1)}$. By Prop. 2, the first condition becomes $\mathfrak{c}=\mathfrak{b}^{k i}$. Thus the second condition becomes $\mathfrak{b}^{k i+1}=\mathfrak{b}^{k(i+1)}$. Since $k>1$, this is equivalent with $\mathfrak{b}^{k i+1}=0$.

Theorem 5. Let $A$ be a hereditary ring and let a be a two-sided ideal in A such that $\mathfrak{a}^{n}=\mathfrak{a}^{n+1}$ for some integer $n>0$. Then

$$
\text { 1. } \operatorname{gl} \operatorname{dim}(\Lambda / \mathfrak{a}) \leqq 2 n-1 \text {. }
$$

Proof. Let $\mathfrak{b}$ be any left ideal in $A$ containing $a$. Since in the projective resolution (1) of $\mathfrak{b} / \mathfrak{a}$ the term $\mathfrak{a}^{n} / \mathfrak{a}^{n+1}$ occuring in degree $2 n-1$ is zero, it follows that

$$
\text { 1. } \operatorname{dim}_{\Delta / \mathfrak{a}}(\mathfrak{b} / \mathfrak{a}) \leqq 2 n-2 .
$$

Since $b / a$ is the most general left ideal in $1 / a$ it follows from a theorem of Auslander [1] that 1.gl. $\operatorname{dim}(\Lambda / \mathfrak{a}) \leqq 2 n-1$. 
COROllary 6 . Let $A$ be a hereditary ring satisfying minimum condition for two-sided ideals. Then for any two-side ideal a in 1

$$
\text { 1. gl. } \operatorname{dim}(A / \mathfrak{a})<\infty \text {. }
$$

\section{$\S 2$. Residue rings of hereditary semi-primary rings}

Proposition 7. Let 1 be a semi-primary ring and a a left ideal in 1. For $n$ sufficiently large we have $a^{n}=a^{n+1}$. Denoting $\bar{a}=a^{n}$ for $n$ sufficiently large we have $(\bar{a})^{2}=\bar{u}$ and

$$
\mathfrak{a}^{k}=\bar{a}+(\mathfrak{a} \cap N)^{k}, \quad k=1,2, \ldots
$$

Proof. Since $A$ is semi-primary there exists an idempotent $e$ such that

$$
\dot{a}=A e+b, \quad \mathfrak{b}=a \cap N .
$$

We assert that

$$
\mathfrak{a}^{k}=\Lambda e \mathfrak{a}+\mathfrak{b}^{k}, \quad k=1,2, \ldots
$$

For $k=1$ we have

$$
\Lambda e \mathfrak{a}+\mathfrak{b}=\Lambda e \Lambda e+\Lambda e \mathfrak{b}+\mathfrak{b}=\Lambda e+\mathfrak{b}=\mathfrak{a} .
$$

Arguing by induction we find

$$
\mathfrak{a}^{k+1}=a a^{k}=\Lambda e \Lambda e a+\Lambda e b^{k}+b \Lambda e a+b^{k+1}=\Lambda e a+b^{k+1} .
$$

Relation (2) implies $\mathfrak{a}^{n}=\mathfrak{a}^{n+1}$ for $n$ large, since $\mathfrak{b}$ is nilpotent. It follows that $\bar{a}=s e a$ and the proof is complete.

THEOREM 8. Let $A$ be a hereditary semi-primary ring. Then for any twosided ideal a in 1 we have

$$
\operatorname{gl} \operatorname{dim}(\Lambda / \mathfrak{a})<\infty \text {. }
$$

This is an immediate consequence of Theorem 5 and Prop. 7. More precise results concerning $\mathrm{gl} \operatorname{dim}(1 / \mathfrak{a})$ will be given in this section.

Consider the representation $a=\bar{a}+(a \cap N), \bar{a}^{2}=\bar{a}$ given in Prop. 7. It follows from Theorem 5 that the ring $A / \bar{a}$ is still hereditary. Thus if we replace $A$ by $A / \bar{a}$ and $\mathfrak{a}$ by $\mathfrak{a} / \bar{a}$, the problem is reduced to the case $a \subset N$ where $N$ is the readical of $A$. This reduction however does not seem to simplify the discussion materially. 
Proposition 9. Let 1 be a hereditary semi-primary ring and let a be a two-sided ideal in .1. The following conditions are equivalnet (for $i \geqq 0)$ :

$$
\begin{gathered}
\text { gl. } \operatorname{dim}(A / \mathfrak{a}) \leqq 2 i+1, \\
\mathfrak{a}^{i}(\mathfrak{a}+N) / \mathfrak{a}^{i+1} \text { is }(A / \mathfrak{a}) \text {-projective, }
\end{gathered}
$$

(iii) there exists a left ideal c in 1 such that

$$
c+a^{i+1}=a^{i}(a+N), \quad c \cap a^{i+1}=a^{i+1}(a+N) .
$$

Similarly the following conditions are equivalent (for $i \geqslant 0$ ):

$$
\text { gl. } \operatorname{dim}(.1 / \pi) \leqq 2 i
$$

$$
a^{i} / a^{i}(a+N) \text { is }(.1 / a) \text {-projective, }
$$

(iii') there exists a left ideal $c$ in 1 such that

$$
\mathfrak{c}+\mathfrak{a}^{i}(\mathfrak{a}+N)=\mathfrak{a}^{i}, \quad \mathfrak{c} \cap \mathfrak{a}^{i}(\mathfrak{a}+N)=\mathfrak{a}^{i+1} .
$$

Proof. Let $A^{\prime}=A /$ a. The radical of $A^{\prime}$ is then $N^{\prime}=(a+N) / a$. Thus

$$
\text { gl. } \operatorname{dim} 1^{\prime}=1+1 \cdot \operatorname{dim}_{\Lambda^{\prime}} N^{\prime}=1+1 \cdot \operatorname{dim}_{\Lambda / \mathfrak{a}}(\mathfrak{a}+N) / \mathfrak{a} .
$$

The result now follows from Prop. 3 applied to the ideal $\mathfrak{b}=\mathfrak{a}+N$.

Remark. The last condition in (iii') implies $a^{i+1} \subset c$. Thus the conditions in (iii') may be rewritten as follows

$$
c+a^{i} N=a^{i}, \quad c \cap a^{i} N \subset a^{i+1} .
$$

Iterating the first condition of (3) we find $c+c N+a^{i} N^{2}=a^{i}$. Further iteration yields $\mathfrak{c}+\mathfrak{c} N+\mathfrak{a}^{i} N^{k}=\mathfrak{a}^{i}$ and thus $\mathfrak{c}+\mathfrak{c} N=\mathfrak{a}^{i}$. In particular, $\mathfrak{a}^{i} N=\mathfrak{c} N$ and may be rewritten as

$$
c+c N=a^{i}, \quad c \cap i N \subset a^{i+1} .
$$

If further aNCNa then $a^{i} N \subset N a^{i}$, and, by Prop. 2, the first condition of (3) becomes $c=a^{i}$. The second condition becomes $a^{i} N \subset a^{i+1}$. Thus in the case $\mathfrak{a} N \subset N \mathfrak{a}$ we find that for $i \geqq 0 \mathrm{gl} \operatorname{dim}(1 / \mathfrak{a}) \leqq 2 i$ is equivalent with

$$
\mathfrak{a}^{i} N \subset \mathfrak{a}^{i+1} .
$$

Proposition 10. Let $A$ be a hereditary semi-primary ring and let a be a

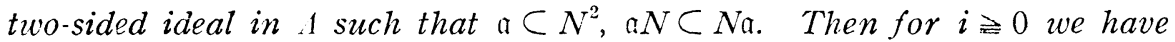

$$
\begin{aligned}
& \text { gl. } \operatorname{dim}(1 / a) \leqq 2 i+1 \Leftrightarrow a^{i+1}=0, \\
& \text { gl. } \operatorname{dim}(1 / a) \leqq 2 i \Leftrightarrow a^{i} N=0 .
\end{aligned}
$$

Proof. The conditions stated in (iii) of Prop. 9 read 


$$
c+a^{i+1}=a^{i} N, \quad c \cap a^{i+1}=a^{i+1} N .
$$

Since $a^{i+1}=a^{i} a \subset a^{i} N^{2} \subset N a^{i} N$, it follows from Prop. 2 that the first condition becomes $\mathfrak{c}=\mathfrak{a}^{i} N$. Since $\mathfrak{a}^{i+1} \subset \mathfrak{a}^{i} N \subset \mathfrak{c}$, the second condition becomes $\mathfrak{a}^{i+1}=\mathfrak{a}^{i+1} N$. This is equivalent with $a^{i+1}=0$.

For the second part we may use the simpler condition ( iii $\left.^{\prime \prime}\right)$. The relation $a^{i} N \subset a^{i+1}$ implies $a^{i} N \subset a^{i} a \subset a^{i} N^{2}$ and this is equivalent with $a^{i} N=0$.

Remark. In the case $i=0$, the condition aNCNa is not needed.

Corollary 11. Let $A$ be a hereditary semi-primary ring. Then, for $n \geqslant 0$,

$$
\text { gl. } \operatorname{dim}\left(A / N^{2}\right)=n \Leftrightarrow N^{n+1}=0, \quad N^{n} \neq 0 .
$$

\section{§. Triangular matrices}

Proposition 12. Consider the ring $A=T_{n+1}(K)$ of all triangular matrices of degree $n+1$

$$
a\left(\alpha_{i j}\right)=\left|\begin{array}{cccc}
\alpha_{n 0}, & 0, & \ldots & 0 \\
\alpha_{10}, & \alpha_{11}, & \ldots & 0 \\
\ldots & \ldots & \ldots & 0 \\
\alpha_{n 0}, & \alpha_{n, 1}, \ldots & \alpha_{n n}
\end{array}\right|
$$

with coefficients in a semi-simple ring $K$. Then 1 is semi-primary, hereditary (gl. $\operatorname{dim} A=1)$ and $\mathrm{gl} \operatorname{dim}\left(\Lambda / N^{2}\right)=n$, where $N$ is the radical of $A$.

Proof. Consider the elements $e, c \in \Lambda$ defined by

$$
\begin{aligned}
& e=a\left(\alpha_{00}=1, \alpha_{i j}=0 \text { for }(i, j) \neq(0,0)\right), \\
& c=a\left(\alpha_{i+1, i}=1, \alpha_{i j}=0 \text { for } i \neq j+1\right) .
\end{aligned}
$$

We obtain an exact sequence

$$
0 \longrightarrow \Lambda \boldsymbol{\varphi} \stackrel{\psi}{\longrightarrow} \Lambda \stackrel{\oplus}{\longrightarrow} N \longrightarrow 0
$$

where $\psi$ is the inclusion while $\varphi \lambda=\lambda c(\lambda \in A)$. Since $e$ is an idempotent $A e$ is a direct summand. Thus the exact sequence splits and therefore $N$ is 1 -projective. Since $N \neq 0$ we have $1 \cdot \operatorname{dim}_{\mathrm{A}} N=0$ so that $\mathrm{gl} \cdot \operatorname{dim} \alpha=1$. The assertion gl. $\operatorname{dim}\left(A / N^{2}\right)=n$ now follows from Cor. 11 since $N^{n} \neq 0=N^{n+1}$.

Proposition 13. Let $A$ be any ring, $A$ a left A-module and let

$$
\ldots \rightarrow 0 \longrightarrow X_{n} \stackrel{d_{n}}{\longrightarrow} X_{n-1} \stackrel{d_{n-1}}{\longrightarrow} \ldots \stackrel{d_{1}}{\longrightarrow} X_{0} \stackrel{d_{0}}{\longrightarrow} A \rightarrow 0
$$


be a projective resolution of $A(n \leqq \infty)$ such that $d_{i} \neq 0$ and $X_{i}$ is indecomposable for all $i \leqq n$. Then $1 \operatorname{dim}_{\mathrm{\Lambda}} A=n$.

Proof. Assume $1 . \operatorname{dim}_{\Lambda} A=k<n$, and denote

$$
Z_{k}=\operatorname{Ker} d_{k}=\operatorname{Im} d_{k+1}
$$

Then $0 \neq Z_{k} \neq X_{k}$. From the exact sequence

$$
0 \longrightarrow X_{k} / Z_{k} \longrightarrow X_{k-1} \longrightarrow \ldots \rightarrow X_{\theta} \longrightarrow A \longrightarrow 0
$$

it follows that $X_{k} / Z_{k}$ is $A$-projective. Therefore the exact sequence

$$
0 \longrightarrow Z_{k} \longrightarrow X_{k} \longrightarrow X_{k} / Z_{k} \longrightarrow 0
$$

splits. This contradicts the indecomposability of $X_{k}$, since $Z_{k} \neq 0 \neq X_{k} / Z_{k}$.

Proposition 14. Consider the ring of all matrices of the form

$$
a(\alpha, \beta, \gamma, \delta, \varepsilon)=\left|\begin{array}{lll}
\alpha & 0 & 0 \\
\gamma & \beta & 0 \\
\varepsilon & \delta & \alpha
\end{array}\right|
$$

with coefficients in a semi-simple ring $K$. Then $A$ is semi-primary, $\operatorname{gl} . \operatorname{dim} A=2$ and $\operatorname{gl} \operatorname{dim}\left(\Lambda / N^{2}\right)=\infty$.

Proof. Consider the elements

$$
\begin{gathered}
e_{1}=a(1,0,0,0,0), \quad e_{2}=a(0,1,0,0,0), \\
c=a(0,0,1,1,0) .
\end{gathered}
$$

Clearly $e_{1}$ and $e_{2}$ are orthogonal primitive idempotents and $e_{1}+e_{2}=1$. We obtain an isomorphism

$$
\theta: A e_{2} \approx N e_{1}
$$

by setting $\theta e_{2}=c e_{1}$; this shows that $N e_{1}$ is projective and indecomposable. Further we obtain an exact sequence

$$
0 \longrightarrow N e_{1} \stackrel{\psi}{\longrightarrow} A e_{1} \stackrel{\rho}{\longrightarrow} N e_{2} \longrightarrow 0
$$

where $\psi$ is the inclusion while $\varphi e_{1}=c e_{2}$. Since both $N e_{1}$ and $A e_{1}$ are indecomposable and projective and $\psi \neq 0 \neq \varphi$ it follows from Prop. 13 that $1 \operatorname{dim}_{\mathrm{A}} N e_{2}$ $=1$. Since $N=N e_{1}+N e_{2}$ is a direct sum, we obtain $1 \cdot \operatorname{dim}_{\mathrm{A}} N=1$ so that gl. $\operatorname{dim} \Lambda=2$.

Now set $A^{\prime}=A / N^{2}$ and let $e_{1}^{\prime}, e_{2}^{\prime}, c^{\prime}$ denote the images of $e_{1}, e_{2}, c$ in $A^{\prime}$. 
Then $e_{1}^{\prime}$ and $e_{2}^{\prime}$ are still orthogonal primitive idempotents in $A^{\prime}$ with $e_{1}^{\prime}+e_{2}^{\prime}=1$. We obtain exact sequences

$$
0 \longrightarrow N^{\prime} e_{2}^{\prime} \stackrel{\psi_{1}}{\longrightarrow} \Lambda^{\prime} e_{2}^{\prime} \stackrel{\varphi_{1}}{\longrightarrow} N^{\prime} e_{1}^{\prime} \longrightarrow 0, \quad 0 \longrightarrow N^{\prime} e_{1}^{\prime} \stackrel{\psi_{2}}{\longrightarrow} \Lambda^{\prime} e_{1}^{\prime} \stackrel{\varphi_{2}}{\longrightarrow} N^{\prime} e_{2}^{\prime} \longrightarrow 0
$$

where $\psi_{1}$ and $\psi_{2}$ are inclusion while $\varphi_{1} e_{2}^{\prime}=c e_{1}^{\prime}, \varphi_{2} e_{1}^{\prime}=c e_{2}^{\prime}$. By composition we obtain a projective resolution

$$
\ldots \longrightarrow \Lambda^{\prime} e_{2}^{\prime} \longrightarrow \Lambda^{\prime} e_{1}^{\prime} \longrightarrow \Lambda^{\prime} e_{2}^{\prime} \longrightarrow \ldots \rightarrow \Lambda^{\prime} e_{1}^{\prime} \longrightarrow \Lambda^{\prime} e_{2}^{\prime} \longrightarrow N^{\prime} e_{1}^{\prime} \longrightarrow 0
$$

where none of the maps is zero. Again by Prop. 13 we have $1 . \operatorname{dim}_{\Lambda^{\prime}}\left(N^{\prime} e_{1}^{\prime}\right)=\infty$. It follows that $\mathrm{gl} \cdot \operatorname{dim} \Lambda^{\prime}=\infty$.

Proposition 15. For each pair $(m, n)$ where $0<m \leqq \infty, 0<n \leqq \infty$, $(m, n) \neq(1, \infty)$, there exist a semi-primary ring $A$ and a two-sided ideal a of 1 contained in the radical of $\Lambda$ such that

$$
\text { gl. } \operatorname{dim} A=m, \quad \operatorname{gl} \cdot \operatorname{dim}(\Lambda / \mathfrak{a})=n .
$$

Proof. The case $m=1$ is settled by Prop. 12. Assume $m>1$. We define $A$ as a direct product $A_{1}+A_{2}$ where $A_{1}$ and $A_{2}$ are semi-primary rings such that

$$
\begin{array}{ll}
\text { gl. } \operatorname{dim} \Lambda_{1}=m, & \text { gl. } \operatorname{dim}\left(\Lambda_{1} / N_{1}\right)=0, \\
\text { gl. } \operatorname{dim} \Lambda_{2} \leqq 2, & \text { gl. } \operatorname{dim}\left(\Lambda_{2} / N_{2}^{2}\right)=n .
\end{array}
$$

The existence of such rings follows from Prop. 12 and 14 . Using the general fact that $\mathrm{gl} . \operatorname{dim} A=\sup \left(\mathrm{gl} . \operatorname{dim} A_{1}, \operatorname{gl} . \operatorname{dim} A_{2}\right.$ ) (see [2], Ch. VI, Exer. 8) we find gl. $\operatorname{dim} A=m$. Now consider the two-sided ideal $a=N_{1}+N_{2}^{2} \subset N$. we have

$$
\Lambda / \mathfrak{a}=\Lambda_{1} / N_{1}+\Lambda_{2} / N_{2}^{2}
$$

so that applying the general rule we obtain $\operatorname{gl} \operatorname{dim}(A / \mathfrak{a})=n$.

\section{§ 4. Algebras}

Let $A$ be an algebra over a (commutative) field $K$ such that $(A: K)<\infty$. Then $A$ is a semi-primary ring. Consider the following statements :

$$
\begin{aligned}
& \operatorname{dim} \Lambda<\infty, \\
& \Gamma=\Lambda / N \text { is separable (i.e. } \operatorname{dim} \Gamma=0 \text { ), } \\
& \operatorname{dim} \Lambda=1+1 . \operatorname{dim}_{\Lambda} N=1 \cdot \operatorname{dim}_{\Delta} \Gamma=\text { gl. } \operatorname{dim} \Lambda .
\end{aligned}
$$

The implications

$$
(\mathrm{a}) \Rightarrow(\mathrm{b}) \Rightarrow(\mathrm{c})
$$


have been established in Ikeda-Nagao-Nakayama [5] and Eilenberg [3]. We further note that if ( $b$ ) holds for $A$ then it also holds for $A^{\prime}=A / a$ where $a$ is any two-sided ideal in 1 . Thus also (c) holds for $A^{\prime}$. These remarks imply that if in $\$ 2 A$ is an algebra as above then all the propositions stated hold with gl. $\operatorname{dim} A$ and $g l \cdot \operatorname{dim}(A / a)$ replaced by $\operatorname{dim} 1$ and $\operatorname{dim}(1 / a)$.

In Propositions 12 and 14 of $\$ 3$ we may take $K$ to be the ground field, and then the rings constructed satisfy (b). Thus the conclusions remain valid with gl. dim replaced by dim. This incidentally supplies examples of algebras with $(1: K)<\infty$ and $\operatorname{dim} A=n$ for any $0 \leqq n \leqq \infty ;$ such examples have been given by Rose [6] and Shih [7].

\section{REFERENCES}

[1] M. Auslander, On the dimension of modules and algebras, III, Nagoya Math. J. 9 (1955), 67-77.

[2] H. Cartan-S. Eilenberg, Homological Algebra, Princeton Univ. Press, 1956.

[3] S. Eilenberg, Algebras of cohomologically finite dimension, Comment. Math. Helv., (1954), 310-319.

[4] S. Eilenberg, M. Ikeda, T. Nakayama, On the dimension of modules and algebras, I, Nagoya Math. J. 8 (1955), 49-57.

[5] M. Ikeda, H. Nagao, T. Nakayama, Algebras with vanishing $n$-cohomology groups, Nagoya Math. J. 7 (1954), 115-131.

[6] I. H. Rose, On the cohomology theory of associative algebras, Amer. J. Math. 74 (1952), 531-546.

[7] K. S. Shih, Dissertation Univ. of Illinois, 1953.

\section{Columbia University \\ Osaka City University \\ Nagoya University}

